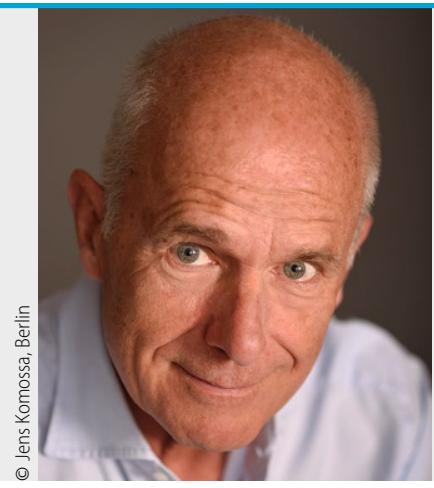

॥) Neurologische Erkrankungen machen auch vor Politikern nicht halt

Prof. Dr. Peter Berlit

Deutsche Gesellschaft für Neurologie (DGN)

Reinhardtstr. 27C, 10117 Berlin, Deutschland

berlit@dgn.org

\title{
Neurologische Erkrankung und Politik
}

Neurologische Erkrankungen sind häufig und betreffen naturgemäß alle Bevölkerungsschichten, ohne vor Personen des öffentlichen Lebens haltzumachen. Auch wenn das öffentliche Interesse bei der Allgegenwärtigkeit sozialer Medien mit Dokumentation etwa von „Zitteranfällen“ der Kanzlerin groß ist, werden in Deutschland Erkrankungen von Politikern - zu Recht - eher als Privatangelegenheit betrachtet.

Anders als in Europa jedoch wird die Gesundheit der amerikanischen Präsidenten von der Öffentlichkeit sehr genau beobachtet und führt zu Änderungen der Verfahrensrichtlinien, sog. „amendments“, mit der Formulierung möglicher Konsequenzen. So gibt es relativ zuverlässige Daten darüber, welche amerikanische Präsidenten neurologisch erkrankt waren.

Bei dem derzeitigen Amtsinhaber wurde beispielsweise recherchiert, ob womöglich Donald J. Trump aufgrund zu geringer Nachtschlafzeit eine chronische Schlafdeprivation mit entsprechenden Auswirkungen auf seine kognitiven Leistungen hat. $\mathrm{Zu}$ diesem Zweck wurde der Twitter-Account des Präsidenten analysiert. Es ergab sich, dass Trump nicht mehr als $5 \mathrm{~h}$ pro Nacht schläft, also sicher weniger als die empfohlenen 7-9 h für Erwachsene [1].

Sowohl Bill Clinton als auch Barack Obama räumten selbst ein, dass sie aufgrund äußerer Umstände häufig zu wenig Nachtschlaf erhielten. Bei Abraham Lincoln ist eine chronische Insomnie beschrieben. William Howard Taft hatte ein Schlafapnoe-Syndrom, bei dem der Body-Mass-Index und damit die Lebensführung sicher eine zentrale Rolle spielten. Taft hatte ein Gewicht von über $150 \mathrm{~kg}$ und fiel während seiner Amtszeit durch Adynamie und Unkonzentriertheit auf [2].

Auch Schmerzen können mit einer deutlichen Beeinträchtigung im Alltag und damit bei verantwortungsvollen Tätigkeiten mit Problemen einhergehen. Unter einer Migräne, welche zumindest tageweise zu deutlichen Einschränkungen führte, litten offensichtlich Thomas Jefferson, der 3. Präsident der USA, und Abraham Lincoln, der 16. Präsident der USA [3]. In einem Brief vom 20.03.1807 notierte Präsident Jefferson:

DGNeurologie 2019 • 2 (5): 333-335

https://doi.org/10.1007/s42451-019-0109-8

๑) Springer Medizin Verlag GmbH, ein Teil von Springer Nature 2019
"Indeed, I have but a little moment in the morning in which I can either read, write, or think; being obliged to be shut up in a dark room from early in the forenoon till night, with a periodical headache" (Zitat aus [3])

Er beschrieb also Symptome, die von Migräne Betroffene kennen und sicher bestätigen, dass während einer solchen Attacke sinnvolle Entscheidungen kaum zu treffen sind.

Bereits in jungen Jahren litt der 35. Präsident der USA, John F. Kennedy, unter chronischen Rückenschmerzen. Insgesamt musste er 4 Rückenoperationen über sich ergehen lassen, u. a. eine Diskektomie und eine Fusionsoperation. Es wird diskutiert, dass diese chronischen Beschwerden durchaus einen Einfluss auf seine Präsidentschaft gehabt haben könnten [4].

Außer Frage steht dieser Zusammenhang bei Schlaganfällen. Insgesamt 11 amerikanische Präsidenten erlitten im Verlauf ihres Lebens einen oder mehrere Schlaganfälle. In Einzelfällen passierte dies auch während der Amtszeit mit entsprechenden Auswirkungen [5]. Rezidivierende Schlaganfälle können ebenso wie eine zerebrale Mikroangiopathie zu einer vaskulären Demenz führen, zu diskutieren bei Woodrow Wilson und Franklin Roosevelt [2].

Das Ende der 2. Amtszeit von Ronald Reagan lag im Jahr 1988. Die Diagnose einer Demenz vom Alzheimer-Typ wurde 1994 offiziell bekannt gegeben. Der größte Skandal der Reagan-Ära, nämlich der Verkauf von Panzerabwehrraketen an den Iran und die anschließende Nutzung der Erlöse für die Finanzierung der rechtsgerichteten Gegner der sandinistischen Regierung in Nicaragua (die sog. Iran-Contra-Affäre) fällt mit einer Krebsoperation bei Reagan im Sommer 1985 zusammen [6]. An die Entscheidungen, die Reagan als Präsident in dieser Zeit fällte, konnte er sich offensichtlich selbst nachher nicht mehr erinnern. Womöglich führte die besondere perioperative Stresssituation zu einer ersten Manifestation der später bestätigten Demenz.

In Deutschland waren die Bundespräsidenten Heinrich Lübke und Walter Scheel von einer Alzheimer-Demenz betroffen [2]. Und es gibt zahlreiche Spekulationen darüber, inwiefern die Parkinson-Erkrankung von Adolf Hitler seine Entscheidungen im zweiten Weltkrieg beeinflusst haben könnte [7].

Bei der legendären Evita kommt ein neurochirurgischer Aspekt mit ins Spiel. Eva Maria Duarte heiratete General Juan Domingo Perón 1946 - in dem Jahr, in dem dieser die Präsidentschaftswahl gewann. Ohne ein offizielles Amt innezuhaben, unterstützte ihn Evita sehr öffentlichkeitswirksam und wurde so 
.......

zur Kultfigur des Peronismus. Im Alter von nur 33 Jahren starb sie 1952 an einem Zervixkarzinom. Mehrere deutsche Spezialisten behandelten sie. Einige Wochen vor ihrem Tod erfolgte eine Lobotomie, und es wird diskutiert, ob diese tatsächlich zur Schmerzbehandlung oder zur Beeinflussung der Persönlichkeit (Psychochirurgie!) erfolgte [8].

In diesem Jahr ist die österreichische neurologische Gesellschaft (ÖGN) auf der Jahrestagung der DGN (Deutsche Gesellschaft für Neurologie) in Stuttgart zu Gast. In einer gemeinsamen Sitzung von DGN und ÖGN werden Referent(inn)en beider Länder das Thema „Neurologische Erkrankung und Politik“ behandeln. Wir dürfen gespannt sein, wie die Sicht der beiden europäischen Nachbarn auf neurologische Erkrankungen mit ihren Auswirkungen auf politische Entscheidungen ausfällt. Seien Sie in Stuttgart dabei!

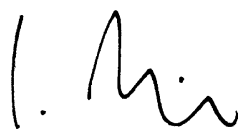

Ihr Peter Berlit

Interessenkonflikt. P. Berlit gibt an, dass kein Interessenkonflikt besteht.
Literatur

1. Kryger M (2017) What can tweets tell us about a person's sleep? J Clin Sleep Med 13(10):1219-1221

2. Förstl H (2018) Demente Staatenlenker: Risiken und Nebenwirkungen. Dtsch Med Wochenschr 143(25):1837-1841

3. Evans RW (2011) Migraine and the presidency. Headache 51:1431-1439

4. Pait TG, Dowdy JT (2017) John F. Kennedy's back: chronic pain, failed surgeries, and the story of its effects on his life and death. J Neurosurg Spine 27(3):247-255

5. Jones $J M$, Jones $J L$ (2006) Presidential stroke: United States presidents and cerebrovascular disease. Cns Spectr 11(9):674-678

6. Gilbert RE (2014) The politics of presidential illness. Ronald Reagan and the IranContra Scandal. Politics Life Sci 33(2):58-76

7. Gupta R, Kim C, Agarwal N, Lieber B, Monaco EA (2015) Understanding the influence of parkinson disease on Adolf Hitler's decision-making during World War II. World Neurosurg 84(5):1447-1452

8. Young GJ, Bi WL, Smith TR, Brewster R, Gormley WB, Dunn IF, Laws ER, Nijensohn DE (2015) Evita's lobotomy. J Clin Neurosci 22(12):1883-1888

\section{Hier steht eine Anzeige.}


Hier steht eine Anzeige.

\section{Springer}

